\title{
Smectic filaments in colloidal suspensions of rods
}

\author{
Daan Frenkel and Tanja Schilling \\ FOM Institute for Atomic and Molecular Physics, Kruislaan 407, 1098 SJ Amsterdam, The Netherlands
}

(Received 4 June 2002; published 28 October 2002)

\begin{abstract}
In supersaturated isotropic mixtures of hard rods, smectic filaments have recently been observed. We propose a model for formation and growth of these filaments similar to the Hoffman-Lauritzen model for polymer crystallization. Filament thickness is determined by a compromise between maximizing the amount of smectic phase formed and minimizing the nucleation barrier for adding new segments to the growing filament. We compare our analytical results to kinetic Monte Carlo simulations.
\end{abstract}

DOI: 10.1103/PhysRevE.66.041606 PACS number(s): 68.70.+w, 64.70.Md, 83.80.Xz, 61.30.Dk

\section{BACKGROUND}

Recent experiments by Dogic and Fraden [1] have shown that thin smectic "filaments" can form in supersaturated mixtures of rodlike virus particles and nonadsorbing polymers. An obvious question is: how are these filaments formed? More specifically, we would like to be able to predict the characteristic dimensions of these filaments.

Below, we shall argue that the mechanism by which the filaments form bears a striking similarity to the HoffmanLauritzen [2] picture of polymer crystallization, i.e., the mechanism of thickness selection is determined by a compromise between maximizing the thermodynamic driving force and minimizing the nucleation barrier for adding new segments to the growing filament.

\section{MODEL}

The basic ingredients of our model are the following: in order to form a smectic phase, the system first has to nucleate a smectic disk with monolayer thickness. However, under the relevant experimental conditions, a single smectic layer is less stable than the metastable isotropic parent phase. Hence, rather than growing in the lateral direction, a second smectic disk will nucleate on top of the first one. Under the appropriate conditions, this can lower the free energy of the smectic aggregate, although many disks may have to be added before the aggregate becomes thermodynamically more stable than the parent phase. Once the growth of a smectic filament has started, it will continue at effectively constant thickness, until the wall of the container is reached, or the concentration of molecules in the parent phase has dropped to a level where further growth and a fortiori further nucleation, is suppressed. This view assumes that the phase transition is of first order. In the present case, the isotropic to smectic transition is coupled to a "vapor-liquid" condensation, and is therefore strongly first order. Under those conditions, the use of a mean-field nucleation picture is not problematic. However, it often happens that one-dimensional ordering transitions (e.g., the formation of lamellar phases) are continuous in the mean-field approximation. The transition is then driven first order by fluctuations [3]. Hohenberg and Swift have shown that, even in this case, it is possible to formulate a self-consistent nucleation theory [4].

Let us consider the mechanism of smectic layer formation in some detail. In the spirit of classical nucleation theory (see e.g., Ref. [5]), we use macroscopic concepts such as bulk densities, surface free energies and chemical potentials to describe the properties of the smectic filaments. We denote the number density of the bulk smectic phase by $\rho$. The difference in chemical potential between the smectic and isotropic phases, $\mu_{S}-\mu_{I}$, is denoted by $\Delta \mu$. As the bulk smectic phase is presumed to be more stable than the isotropic phase, $\Delta \mu<0$. The thickness of a single smectic layer is denoted by $d$. Finally, we assume that the surface free energy of a smectic domain is anisotropic. The surface free energy associated with the interface between the top of a smectic layer and the adjacent isotropic phase, is denoted by $\gamma_{\perp}$, where the symbol $\perp$ is used to indicate that this layer is perpendicular to the molecular axes. The interfacial free energy of the interface between the edges of the smectic layer and the isotropic phase, is denoted by $\gamma_{\|}$. Implicitly, we assume that, for the description of filament formation, it is permissible to ignore the fact that the constituent rodlike viruses are chiral.

With these definitions, we can write down the free energy associated with the formation of a circular smectic disk with radius $R$ :

$$
\Delta F_{0}(R)=-\pi R^{2} \rho d|\Delta \mu|+2 \pi R^{2} \gamma_{\perp}+2 \pi R d \gamma_{\|} .
$$

As the first two terms on the right-hand side of Eq. (1) are proportional to $R^{2}$, we can combine them to obtain

$$
\Delta F_{0}(R)=\pi R^{2}\left(2 \gamma_{\perp}-\rho d|\Delta \mu|\right)+2 \pi R d \gamma_{\|} .
$$

At low supersaturation, when $|\Delta \mu|<2 \gamma_{\perp} /(\rho d), \Delta F_{0}(R)$ is positive. This implies that a single smectic layer cannot grow, even though the bulk smectic phase is more stable than the isotropic phase.

Let us next consider the formation of a second smectic disk, on top of the first one. As long as the surface of this second disk does not exceed that of the original disk, no new "top" surface is created in this process. As a consequence, the free energy needed to grow a second disk of diameter $r$ is equal to

$$
\Delta F_{1}(r)=-\pi r^{2} \rho d|\Delta \mu|+2 \pi r d \gamma_{\|}
$$

For small $r$, this free energy is also positive. However, it goes through a maximum at $r_{\max }=\gamma_{\|} /(\rho|\Delta \mu|)$, and becomes 
negative for $r>2 r_{\max }$. If the radius of the initial disk was larger than $2 r_{\max }$, then addition of a new smectic layer with the same radius $R$ as the first layer lowers the free energy of the aggregate by an amount

$$
\Delta f(R)=-\pi R^{2} \rho d|\Delta \mu|+2 \pi R d \gamma_{\|} .
$$

This process can be repeated several times, and every time the free energy of the smectic "filament" will be lowered by the same amount. Eventually, after

$$
n=\frac{2 R \gamma_{\perp}}{R \rho d|\Delta \mu|-2 d \gamma_{\|}}
$$

layers have been formed, the smectic filament becomes absolutely stable with respect to the isotropic parent phase and will go on growing until stopped by other factors. As the driving force for growth vanishes for disk radii less than $R$ $=2 \gamma_{\|} /(\rho|\Delta \mu|)$, no filaments with smaller diameter can form. On the other hand, the free energy needed to form the first disk increases monotonically with $R$. Hence the nucleation barrier to form a filament grows rapidly with $R$. The optimum radius for smectic filaments is therefore expected to be only slightly larger than $2 \gamma_{\|} /(\rho|\Delta \mu|)$. The competition between driving force and nucleation rate is also at the root of the Hofmann-Lauritzen model for the layer-by-layer growth of lamellar polymer crystals [2]. In fact, much of that theory can be carried over with minor modifications to the present case.

\section{KINETICS}

The rate of formation of smectic filaments is determined by two factors: the first is the rate of formation of a first disk with radius $R$. The second is the rate of addition of disks on an existing disk with radius $R$. Both processes are activated. That means that we can express the rate of addition of disk $(i+1)$ to a stack of $i$ disks, as

$$
k_{i \rightarrow i+1}^{+}=\Gamma_{i} \exp \left[-\beta \Delta F_{B}(i \rightarrow i+1)\right],
$$

where $\Gamma_{i}$ is a kinetic prefactor. As we shall argue below, this prefactor is independent of $i$. Hence, in what follows, we drop the subscript $i . \Delta F_{B}(i \rightarrow i+1)$ is the free-energy barrier that has to be crossed when going from state $i$ to $i+1$. In fact, only two different barriers need to be distinguished. The first is the barrier that separates the initial isotropic state from a hypothetical state with one smectic disk of radius $R$. We denote this barrier by $\Delta F_{0}(R)$. This state is called a "hypothetical" state, because it does not correspond to a local minimum in the free energy. The first state that does, is the one with two disks. The second relevant free-energy barrier is the one that separates states $i$ and $i+1$, for $i \geqslant 2$. This barrier, we denote by $\Delta F_{1}$ (Fig. 1). The height of this barrier can be derived from Eq. (3),

$$
\Delta F_{1}=\pi \gamma_{\|}^{2} d /(\rho|\Delta \mu|) .
$$

Note that this barrier does not depend on $R$. To compute the rate of formation of filaments, we assume that steady-state

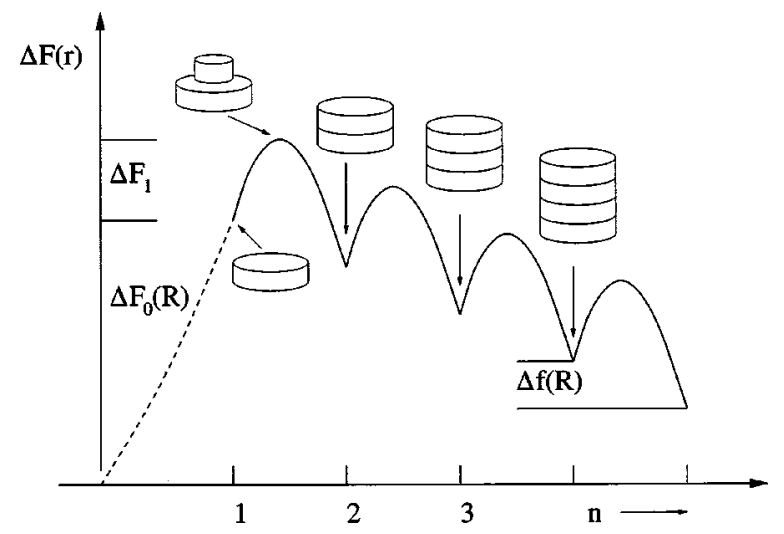

FIG. 1. Nucleation barrier for smectic filaments.

conditions apply. This means that the concentrations of all intermediate species (i.e., those consisting of $2,3,4, \ldots$ disks) are constant in time. For every transition from $i$ to $i$ +1 , there is a reverse transition with a rate constant $k_{i+1 \rightarrow i}^{-}$. If we denote by $I$ the steady-state rate of addition of disks to a filament, then we have the following relation between forward and backward reactions:

$$
N_{i} k_{i \rightarrow i+1}^{+}-N_{i+1} k_{i+1 \rightarrow i}^{-}=I .
$$

Moreover, in equilibrium, $I=0$ and hence we obtain the detailed-balance condition

$$
\frac{N_{i+1}^{0}}{N_{i}^{0}}=\frac{k_{i \rightarrow i+1}^{+}}{k_{i+1 \rightarrow i}^{-}},
$$

where $N_{i}^{0}$ denotes the equilibrium concentration of a filament consisting of $i$ smectic disks of radius $R$. The ratio between the equilibrium concentrations is directly related to the freeenergy difference between states $i+1$ and $i$ :

$$
\frac{N_{i+1}^{0}}{N_{i}^{0}}=\exp \{-\beta[F(i+1)-F(i)]\}
$$

To compute the steady-state rate $I$, we use the standard trick of expressing $I$ as

$$
\begin{aligned}
I & =N_{0} k_{0 \rightarrow 2}^{+}-N_{2} k_{2 \rightarrow 0}^{-}=N_{2} k_{2 \rightarrow 3}^{+}-N_{3} k_{3 \rightarrow 2}^{-} \cdots \\
& =N_{i} k_{i \rightarrow i+1}^{+}-N_{i+1} k_{i+1 \rightarrow i}^{-} .
\end{aligned}
$$

The $i$ th term in the above set of equations can be written as

$$
I=N_{i} k_{i \rightarrow i+1}^{+}-N_{i+1} k_{i+1 \rightarrow i}^{-}=N_{i}^{0} k_{i \rightarrow i+1}^{+}\left(\frac{N_{i}}{N_{i}^{0}}-\frac{N_{i+1}}{N_{i+1}^{0}}\right),
$$

and hence

$$
\frac{N_{i}}{N_{i}^{0}}-\frac{N_{i+1}}{N_{i+1}^{0}}=\frac{I}{N_{i}^{0} k_{i \rightarrow i+1}^{+}} .
$$


We now make use of the fact that $N_{i} \approx N_{i}^{0}$ for $i=0$, indicating that the initial state is in (metastable) equilibrium, while for $i \gg 1,\left(N_{i} / N_{i}^{0}\right) \approx 0$. Then, by summing Eq. (10) for all $i$, we get

$$
1=I\left(\frac{1}{N_{0}^{0} k_{0 \rightarrow 2}^{+}}+\sum_{i=2}^{\infty} \frac{1}{N_{i}^{0} k_{i \rightarrow i+1}^{+}}\right) .
$$

This expression can be simplified by using Eq. (5) for the rate constants to express the ratio

$$
\frac{k_{0 \rightarrow 2}^{+}}{k_{i \rightarrow i+1}^{+}}=\exp \left\{-\beta\left[\Delta F_{0}(R)\right]\right\} .
$$

Using the symbol $k_{1}^{+}$for $k_{i \rightarrow i+1}^{+}$for all $i \geqslant 2$, we can rewrite Eq. (11) as

$$
1=\frac{I}{k_{1}^{+}}\left(\frac{1}{N_{0}^{0} \exp \left\{\beta\left[\Delta F_{0}(R)\right]\right\}}+\sum_{i=2}^{\infty} \frac{1}{N_{i}^{0}}\right) .
$$

We can perform the summation in the second term on the right-hand side, and use Eq. (4) to obtain

$$
\begin{aligned}
1 & =\frac{I}{k_{1}^{+}}\left(\frac{1}{N_{0}^{0} \exp \left\{\beta\left[\Delta F_{0}(R)\right]\right\}}+\sum_{i=2}^{\infty} \frac{1}{N_{i}^{0}}\right) \\
& =\frac{I}{k_{1}^{+} N_{0}^{*}\{1-\exp [\beta \Delta f(R)]\}},
\end{aligned}
$$

where $N_{0}^{*} \equiv N_{0}^{0} \exp \left[-\beta \Delta F_{0}(R)\right]$. The expression we then obtain for the rate of growth of smectic filaments is

$$
I=k_{1}^{+} N_{0}^{*}\{1-\exp [\beta \Delta f(R)]\} .
$$

Using Eq. (5), we can rewrite this as

$$
I=N_{0}^{0} k_{1}^{+} \exp \left\{-\beta\left[\Delta F_{0}(R)\right]\right\}\{1-\exp [\beta \Delta f(R)]\} .
$$

Equation (15) exhibits the behavior mentioned above: it yields a vanishing rate when $\Delta f(R)$ vanishes, i.e., when there is no net thermodynamic driving force for filament formation. And, in addition, $I$ decreases steeply with increasing height of the nucleation barrier.

\section{PREFACTOR}

The rate at which new disks are added to the filament can be estimated, assuming that the addition of a disk involves a diffusive barrier crossing. Let us first compute the rate at which the transition from a state with $i$ disks to one with $i$ +1 disks takes place. This rate is equal to

$$
k_{1}^{+}=\exp \left(-\beta \Delta F_{1}\right) k_{\text {add }} \sqrt{\frac{\left|\Delta F_{1}{ }^{\prime \prime}\right|}{2 \pi}},
$$

where $k_{\text {add }}$ is the rate at which single rods are added to a disk at the top of the nucleation barrier and $\Delta F_{1}{ }^{\prime \prime}$ is the curvature at the top of the free-energy barrier

$$
\Delta F_{1}^{\prime \prime}=\frac{\partial^{2} \Delta F(n)}{\partial n^{2}} .
$$

Note that the variable is not the disk radius $r$, but $n$, the number of rods in a disk with radius $r . n$ is related to $r$ through

$$
n=\rho \pi r^{2} d,
$$

and hence

$$
\Delta F_{1}^{\prime \prime}=-\frac{(\Delta \mu)^{3} \rho}{2 \pi d \gamma_{\|}^{2}} .
$$

Combining these results, we get

$$
k_{1}^{+}=\exp \left[-\beta \pi \gamma_{\|}^{2} d /(\rho \Delta \mu)\right] k_{a d d} \sqrt{\frac{(\Delta \mu)^{3} \rho}{d\left(2 \pi \gamma_{\|}\right)^{2}}} .
$$

We can estimate $k_{\text {add }}$ to be of order

$$
2 \pi r d \rho_{I} D_{\perp} / \lambda,
$$

where $\rho_{I}$ is the number density in the isotropic phase, $D_{\perp}$ is the transverse diffusion constant of the rods, and $\lambda$ is a characteristic diffusion length. The overall rate of growth per unit volume of smectic filaments with radius $R$ is then

$$
\begin{aligned}
\Gamma(R)= & I / V=\rho_{I} \exp \left[-\beta \pi \gamma_{\|}^{2} d /(\rho \Delta \mu)\right] 2 \pi\left(\frac{\gamma_{\|}}{\rho \Delta \mu}\right) d \rho_{N} D_{\perp} / \lambda \\
& \times \sqrt{\frac{(\Delta \mu)^{3} \rho}{d\left(2 \pi \gamma_{\|}\right)^{2}}} \exp \left\{-\beta\left[\Delta F_{0}(R)\right]\right\} \\
& \times\{1-\exp [-\beta \Delta f(R)]\} .
\end{aligned}
$$

This rate still depends on the filament radius. To find the radius of the fastest growing filaments, we should determine the value of $R$ for which $\Gamma(R)$ is maximized.

\section{THERMAL FLUCTUATIONS}

\section{A. Model}

So far we have assumed that a filament grows with a constant radius, once the size of the first disk is established. The model has not taken thermal fluctuations into account. We investigate their influence within a kinetic Monte Carlo simulation.

We start out with a disk of random radius and add or subtract a disk in each time step according to the rates from Eqs. (5) and (7). Given a filament of $i$ disks, a new disk is added with probability 

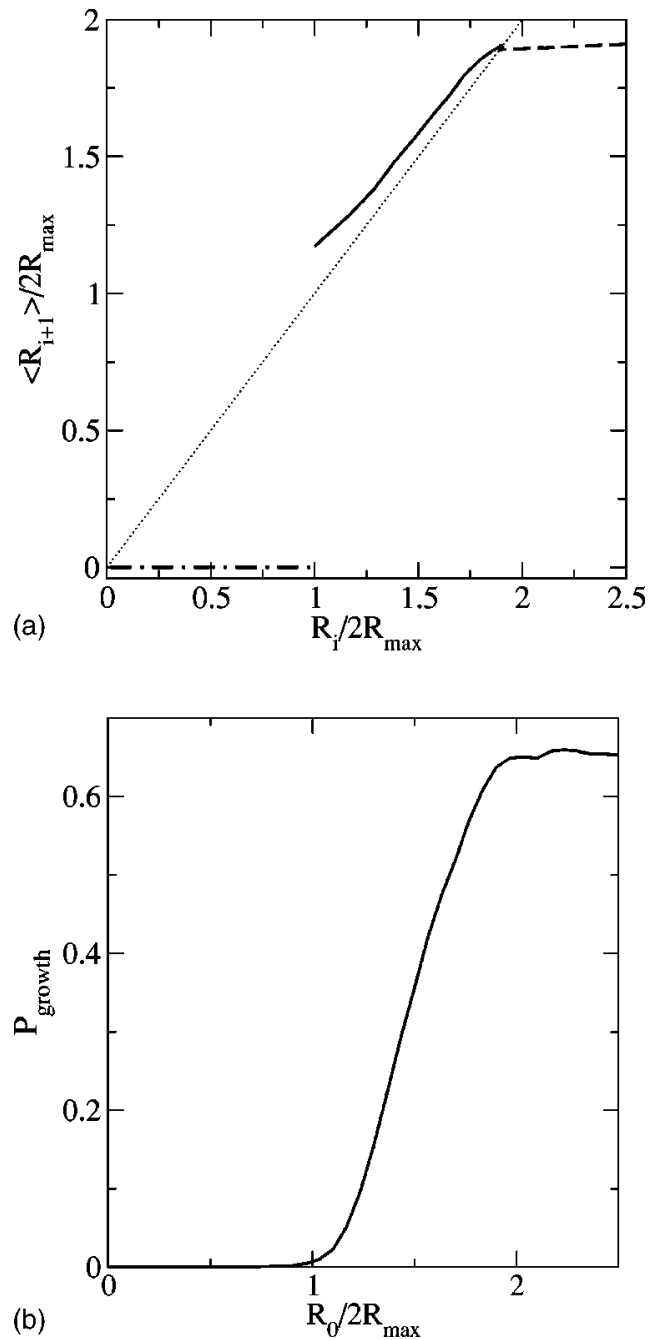

FIG. 2. (a) Most probable radius of disk $(i+1)$ versus radius of disk $i$. For $R_{i}>2 r_{\text {max }}\left\langle R_{i+1}\right\rangle$ goes towards a fixed point at a finite value (solid and dashed line). For smaller $R_{i}$ it goes to zero (dotdashed line). (b) Probability of adding a disk (of any size), which will not be removed again, to a disk of radius $R_{0}$. $\quad\left(\gamma_{\|}=0.7\right.$, $S=0.8$.)

$$
P^{+}\left(R_{i}\right)=\frac{k_{1}^{+}}{k_{1}^{+}+k_{i \rightarrow i-1}^{-}}=\frac{1}{1+\exp \{\beta[F(i)-F(i-1)]\}},
$$

where we have used the fact that the forward rate is independent of $i$ and depends only on the barrier height for the nucleation of a new disk. If no new disk is added the $i$ th disk is removed. The length of a time step $1 /\left(k_{1}^{+}+k_{i \rightarrow i-1}^{-}\right)$depends on the free-energy difference between the last two disks.

The radius of the added disk $R_{i+1}$ is sampled from a Boltzmann distribution with respect to the change in free energy it produces

$$
\begin{aligned}
P\left(R_{i+1}\right)= & \exp \left\{-\beta\left[-\pi R_{i+1}^{2} \rho d|\Delta \mu|+2 \pi R_{i+1} d \gamma_{\|}\right.\right. \\
& \left.\left.+\Theta\left(R_{i+1}-R_{i}\right) 2 \pi\left(R_{i+1}^{2}-R_{i}^{2}\right) \gamma_{\perp}\right]\right\},
\end{aligned}
$$

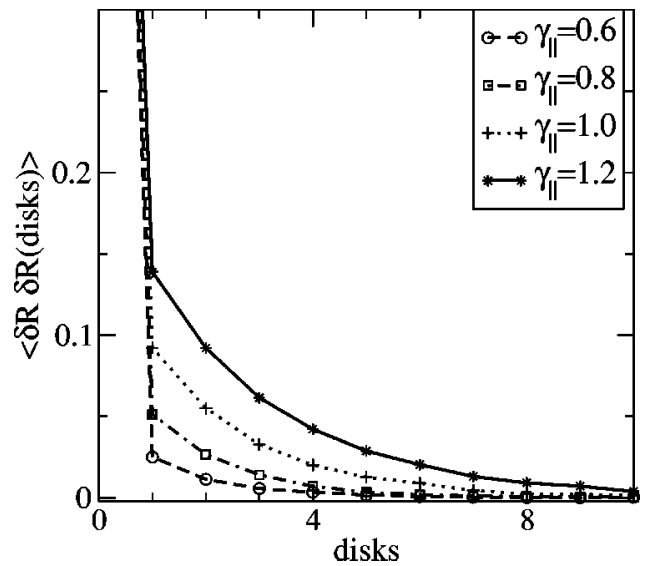

FIG. 3. Correlation of radii along the filament. Radii are correlated over a few disks. The correlation becomes longer with growing $\gamma_{\|}(S=0.9)$.

where the $\Theta$ function takes into account the production of additional surface if a disk is larger than the previous. The parameters of the model are $\rho, \Delta \mu, d, \gamma_{\perp}, \gamma_{\|}$, and $\beta$. In the following discussion of the results $d=\gamma_{\perp}=\beta=1$, and we combine all other parameters into the supersaturation $S$ $=2 \gamma_{\perp} / \rho d \Delta \mu$.

\section{B. Results}

In the simulation the average radius of a filament differs from the radius of its first disk. If the radius of the first disk is larger than $2 r_{\max }$ a filament adjusts its average radius to one fixed value $R_{s}$. Otherwise it shrinks to zero. In Fig. 2(a) the most probable radius of disk $(i+1)$ is plotted against the radius of the $i$ th disk. Starting out from radii larger than $2 r_{\max }$ (solid and dashed line) all paths end in a fixed point at $R_{s}$. Starting out from smaller radii (dot-dashed line) all paths go to zero. Figure 2(b) shows the probability of producing a disk of any size which will not be removed again on top of a disk of size $R_{0}$. It is almost zero for radii smaller than $2 r_{\max }$ and grows to reach a plateau at the fixed point.

Figure 3 shows the correlation of radii along the filament (normalized to 1 at disk 0) for several values of $\gamma_{\|}$. Radii are correlated over a few disks, and the correlation length grows with increasing $\gamma_{\|}$and with increasing supersaturation.

The analytical expression for the growth rate $I(R)$, Eq. (15), has a maximum at a radius $R_{\max I}$. We find $R_{s}$ to be larger than $R_{\text {max }}$. This is due to the asymmetry of the free energy in Eq. (18), which on average causes disks of a radius larger than $2 r_{\max }$ to be added. The rate $I\left(R_{s}\right)$ at which filaments grow in the simulation can be compared to the analytical rate $I(R)$ from Eq. (15) if the probablity to form the starting disk $P\left(R_{0}=R\right)$, and the probability to grow a stable filament from it $P\left(R \rightarrow R_{s}\right)$ are included,

$$
I_{\text {sim }}(R)=P\left(R_{0}=R\right) P\left(R \rightarrow R_{s}\right) I\left(R_{s}\right) .
$$

We find $I_{\text {sim }}\left(R_{\text {maxI }}\right)$ to be one to two orders of magnitude smaller than $I\left(R_{\max I}\right)$ because of thermal fluctuations. 
Thermal fluctuations stabilize the growth of filaments of one fixed average thickness. This thickness only depends on the system parameters such as surface tensions and supersaturation, but not on the thickness of the first disk. The average thickness is larger than the thickness of fastest growth from the analytical analysis and the filaments grow more slowly. Disk size is correlated over a few disks.

\section{CONCLUSIONS}

In this paper we have proposed a model for formation and growth of smectic filaments in isotropic solutions of hard rods. (The formation in a nematic solution can be described in a similar way). As in the Hoffman-Lauritzen approach the basic mechanism behind the formation process is the minimization of the nucleation barrier for adding new layers. We have predicted radii and growth rates.
We have performed kinetic Monte Carlo simulations in order to take into account thermal fluctuations. Thermal fluctuations decrease the growth rate and stabilize the growth of filaments of one fixed average thickness regardless of the sizes of their first disks.

\section{ACKNOWLEDGMENTS}

We thank Zvonimir Dogic for helpful discussions. This work was part of the research program of "Stichting Fundamenteel Onderzoek der Materie (FOM)" which was financially supported by "Nederlandse Organisatie voor Wetenschapelijke Onderzoek (NWO)." T.S. acknowledges financial support through the Fifth Framework Program of the European Commission.
[1] Z. Dogic and S. Fraden, Philos. Trans. R. Soc. London, Ser. A 359, 997 (2001).

[2] J.I. Lauritzen and J.D. Hoffman, J. Appl. Phys. 44, 4340 (1973).

[3] S.A. Brazovskii, Zh. Eksp. Teor. Fiz. 68, 175 (1975) [Sov.
Phys. JETP 41, 85 (1975)].

[4] P.C. Hohenberg and J.B. Swift, Phys. Rev. E 52, 1828 (1995).

[5] K.F. Kelton, Solid State Physics (Academic Press, San Diego, 1991), Vol. 45, p. 75. 